\title{
L'uso ospedaliero di ciprofloxacina nella terapia parenterale ed orale delle infezioni gravi: profilo farmacoeconomico
}

Mario Eandi*, Carlo Della Pepa*

\begin{abstract}
The study focuses on the microbiological and economic aspects of the treatment of hospital infections. The following topics are presented and discussed: I) principal microbiological features of infections acquired during hospital stays; II) data on the use of fluoroquinolones in hospital patients; III) cost-analysis of hospital infection treatment; IV) impact of different antibiotics on the selection of resistant strains of Pseudomonas aeruginosa; V) review of pharmacoeconomic data of different antibacterial regimens.
\end{abstract}

Farmeconomia e percorsi terapeutici 2001; 2 (4): 201-216

\section{INTRODUZIONE}

Tra i derivati fluorochinolonici attualmente più utilizzati nel trattamento delle infezioni in ambito ospedaliero vi sono la ciprofloxacina e la levofloxacina ${ }^{1}$. La ciprofloxacina, chinolonico di terza generazione, short-acting a spettro amplissimo, è considerata ancora oggi il fluorochinolone di riferimento. Lo spettro d'azione, infatti, risulta essere il più ampio sia tra i fluorochinoloni sia, praticamente, tra tutti gli antibatterici di uso clinico ${ }^{2}$.

La levofloxacina, isomero levogiro di ofloxacina, viene classificato come chinolonico di terza generazione, short-acting, a spettro ampio $^{2}$.

Rispetto alla ciprofloxacina, infatti, pur presentando una migliore attività nei confronti di alcuni Gram-positivi (pneumococchi, stafilococchi meticillino sensibili, streptococchi), presenta un'attività inferiore verso alcuni Gram-negativi (Enterobacteriaceae, Pseudomonas aeruginosa $)^{2}$.

Le politiche di contenimento dei costi per le prestazioni sanitarie, comuni da alcuni anni in tutti i Paesi occidentali, hanno obbligato a considerare attentamente le spese determinate dall'approvvigionamento ospedaliero dei farmaci, spese che vedono negli antibatterici una voce di considerevole entità.

La diversa attività antibatterica di ciprofloxacina e di levofloxacina e le differenze nel profilo clinico-terapeutico depongono sfavorevolmente per la sostituibilità tra un derivato fluorochinolonico e l'altro basata semplicemente sul costo giornaliero della terapia. Prima di procedere così semplicisticamente è bene valutare quanto pubblicato in letteratura nello specifico ambito, cioè nelle indagini farmacoeconomiche; esse, infatti, danno una valutazione non solo del prezzo da pagare per il farmaco, ma dei costi globali a carico dell'ente che eroga il servizio "Salute", in funzione di un determinato regime terapeutico. Il farmaco, infatti, in molti casi incide solo marginalmente nel costo totale di gestione di una patologia.

Una considerazione indicativa a sostegno di quest'ultima asserzione fa riferimento all'introduzione dei fluorochinoloni in terapia. Questi farmaci hanno consentito l'attuazione pratica efficace e sicura della terapia sequenziale ${ }^{3}$ (trattamento e.v. in prima istanza seguito dalla somministrazione orale dello stesso principio attivo). L'elevata attività antibatterica e le caratteristiche farmacocinetiche particolarmente favorevoli di diffusibilità tessutale dei fluorochinoloni somministrati per $o s$ hanno reso possibile questa strategia terapeutica. In questo modo si sono ridotti considerevolmente $i$ costi sanitari sia per la riduzione delle giornate di degenza (minori costi sanitari diretti) sia per il precoce allontanamento del paziente da un ambiente, quello ospedaliero, a elevato potenziale di contagio (prevenzione di patologie in- 
fettive il cui trattamento comporta oneri economici).

Un importante aspetto da considerare, quando si valuta la sostituibilità di un fluorochinolonico con un altro, è legato alle modificazioni della flora batterica indotte in ospedale qualora si sostituisca un derivato altamente efficace (ciprofloxacina) verso ceppi ad alta morbilità ospedaliera (Pseudomonas aeruginosa ad esempio) con un fluorochinolonico meno attivo. Selezionare ceppi di Pseudomonas aeruginosa resistenti ai fluorochinoloni può comportare problemi, e non solo economici, di portata davvero considerevole.

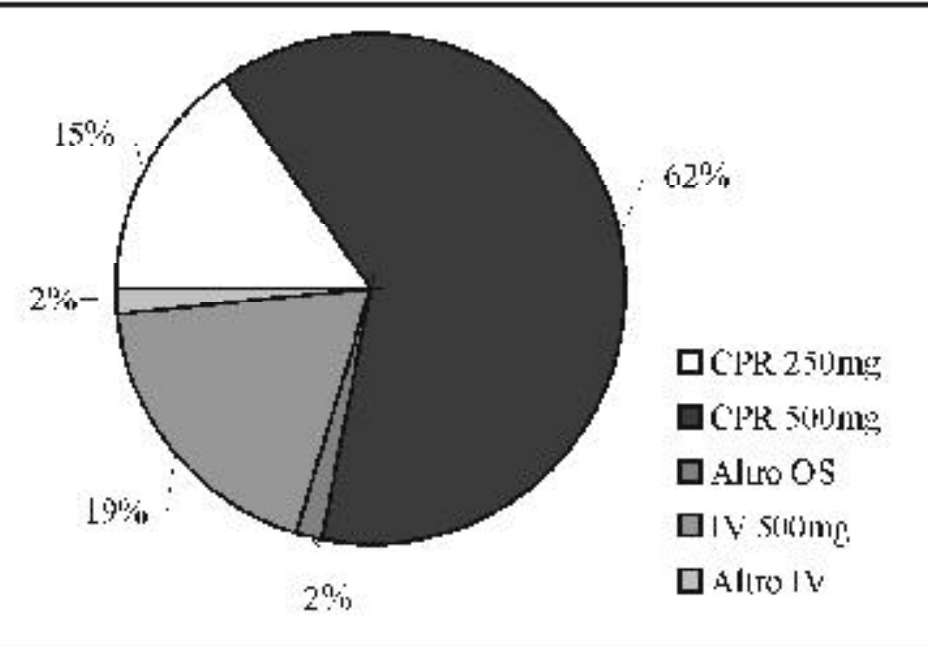

Figura 1

Distribuzione percentuale del numero dei trattamenti effettuati durante l'anno 2000 negli ospedali italiani con le diverse forme di ciprofloxacina: "altro" indica i casi in cui il trattamento è stato effettuato con più forme farmaceutiche (Elaborato da dati AMR, $2000^{I}$ ).

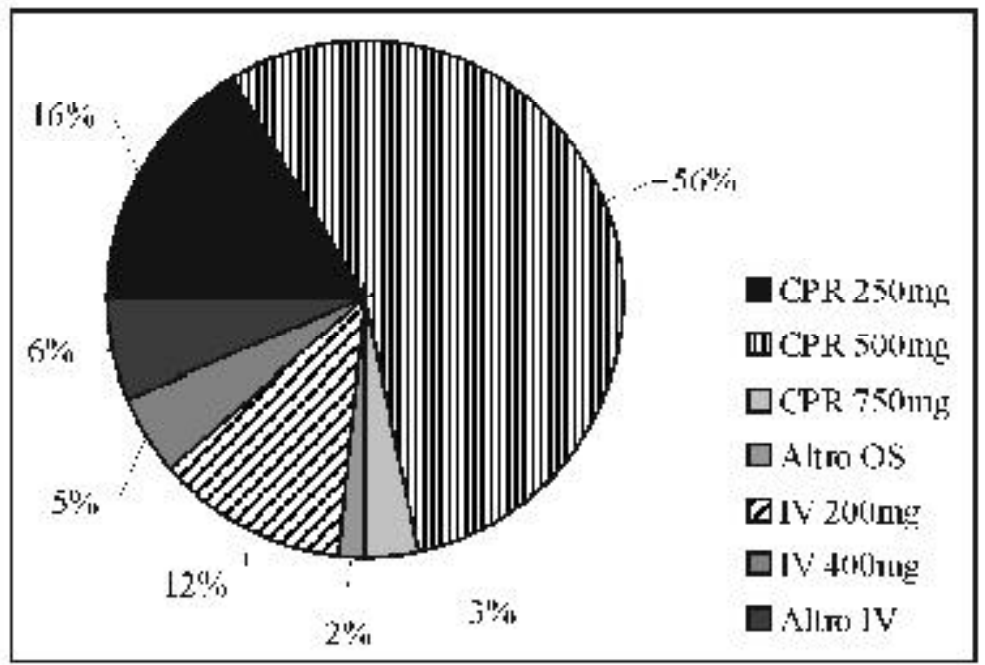

Figura 2.

Distribuzione percentuale del numero dei trattamenti effettuati durante l'anno 2000 negli ospedali italiani con le diverse forme di levofloxacina: "altro" indica $i$ casi in cui il trattamento è stato effettuato con più forme farmaceutiche (Elaborato da dati AMR, 2000').

\section{MICROBIOLOGIA}

Negli ultimi anni, uno degli aspetti più allarmanti della microbiologia clinica è stato l'elevato aumento di incidenza di resistenze agli antibatterici tra i patogeni responsabili di infezioni sia nosocomiali, sia comunitarie. Numerosi antibiotici hanno perso la loro attività, come dimostrato da studi in vitro, quale risultato della pressione selettiva esercitata dall'uso degli antibatterici stessí. Per fronteggiare questa emergenza, sono in essere numerosi progetti di ricerca: Alexander Project ${ }^{5}$ in Europa e Nordamerica, SENTRY Programme ${ }^{6}$ in Europa, Osservatorio Epidemiologico Italiano ${ }^{7}$ in Italia.

Anche se l'aspetto relativo alla presenza di patogeni contaminanti è strettamente struttura-specifico, riportiamo qui di seguito dei dati relativi a realtà italiane e internazionali.

Una recente indagine condotta presso un ospedale italiano dal laboratorio di microbiologia e virologia ${ }^{8}$ in pazienti ricoverati in Unità di Terapia Intensiva (UTI), in degenza ordinaria, e pazienti ambulatoriali afferenti all'ospedale stesso ha evidenziato che i patogeni isolati con maggiore frequenza erano, tra $\mathrm{i}$ Grampositivi Staphylococcus aureuse stafilococchi coagulasi negativi; tra i Gram-negativi Enterococcus spp., Pseudomonas aeruginosa e Escherichia coli. Resistenze alle penicilline e alle cefalosporine di prima generazione erano osservate più frequentemente nei pazienti ricoverati in UTI, quindi in quelli in ricovero ordinario e, infine, tra i pazienti ambulatoriali. Pseudomonas aeruginosa risultava avere un basso tasso di sensibilità a cefalosporine di II, III e IV generazione, carbapenemi e monobattami, soprattutto tra i pazienti in degenza ordinaria. Risultavano sensibili a ciprofloxacina i ceppi di $P$. aeruginosa isolati in UTI.

I dati di questa indagine italiana sono confermati su base internazionale relativamente alla flora batterica responsabile di infezioni nosocomiali. Un recente studio statunitense ${ }^{9}$ conferma come Staphylococcus aureus (compresi i ceppi meticillino-resistenti), enterococchi, Escherichia coli e Pseudomonas aeruginosa siano i batteri più frequentemente responsabili di infezioni nosocomiali.

In questo contesto, vale la pena di ribadire che, da un punto di vista microbiologico, levofloxacina presenta attività più spiccata verso i Gram-positivi e più limitata verso i Gramnegativi, in particolare verso $P$. aeruginosa ${ }^{2}$; ciprofloxacina è ancora considerata il fluorochinolone di riferimento perché caratterizzata dallo spettro d'azione più ampio conosciuto finora che la posiziona indiscutibilmente come farmaco di scelta nelle infezioni nosocomiali, in particolare quando presente Pseudomonas aeruginosa ${ }^{2}$. 
UTILIZZAZIONE DI CIPROFLOXACINA E levofloxacina viene utilizzata con frequenza reLEVOFLOXACINA NEGLI OSPEDALI ITA-

\section{LIANI}

Un campione significativo di casi trattati con ciprofloxacina oppure con levofloxacina negli ospedali italiani durante l'anno 2000 è stato rilevato dall'agenzia AMR (The Hospital AntiInfective Market).

Il campionamento è stato predisposto considerando la stratificazione della casistica nazionale per aree geografiche, per dimensioni e tipologie dell'ospedale, nonché per specialità delle divisioni ospedaliere. La dimensione del campione, per ogni stratificazione, rappresenta circa il $10-13 \%$.

I dati campionati, dopo essere stati sottoposti a rigorosi controlli formali e sostanziali, sono stati estrapolati per stimare in proiezione nazionale la utilizzazione ospedaliera dei due fluorochinoloni nelle diverse divisioni specialistiche ospedaliere.

La proiezione su scala nazionale annua è stata attuata secondo procedure statistiche consolidate e validate ${ }^{10}$.

In base a questa indagine si stima che durante l'anno 2000 negli ospedali italiani siano stati effettuati circa 293.000 trattamenti con ciprofloxacina, dei quali circa 225.000 per via orale e 68.000 per via parenterale. La levofloxacina è stato utilizzata complessivamente in circa 59.000 trattamenti, 47.000 per via orale e 12.000 per via parenterale.

Le Figure 1 e 2 riportano le ripartizioni percentuali del numero di trattamenti effettuati rispettivamente con ciprofloxacina e con levofloxacina, suddivisi in base alla forma farmaceutica utilizzata ed al livello dose.

Entrambi i fluorochinoloni sono utilizzati prevalentemente per via orale, la ciprofloxacina nel $77 \%$ e la levofloxacina nel $79 \%$ dei trattamenti. Le compresse da $250 \mathrm{mg}$ sia di ciprofloxacina sia di levofloxacina sono utilizzate in circa il $15 \%$ dei casi, mentre la compressa da $500 \mathrm{mg}$ è usata in circa il $60 \%$ dei casi. Nell'ambito della via parenterale la ciprofloxacina viene utilizzata prevalentemente in flaconi da $200 \mathrm{mg}$. La levofloxacina parenterale è disponibile in un'unica posologia di $500 \mathrm{mg}$.

Le Figure 3 e 4 riportano le distribuzioni del numero di trattamenti effettuati rispettivamente con ciprofloxacina e levofloxacina nelle varie divisioni ospedaliere.

Entrambi i fluorochinoloni risultano essere utilizzati prevalentemente nei reparti di Medicina Interna, seguiti a distanza dai reparti di Chirurgia Generale e di Infettivologia. Confrontando le rispettive distribuzioni, ciprofloxacina, a differenza della levofloxacina, viene utilizzata con frequenza relativamente elevata anche nei reparti di Urologia, mentre, al contrario, lativamente elevata anche nei reparti di Pneumologia e di Ginecologia. Levofloxacina non risulta praticamente utilizzata nei reparti di Chirurgia Ortopedica, Dermatologia e Geriatria.

Le giornate cumulative di terapia con ciprofloxacina sono state 1.720 .000 , delle quali circa 3/4 dovute a trattamenti per via orale e solo $1 / 4$ a trattamenti per via parenterale. Le giornate cumulative di terapia con levofloxacina sono state 346.000 , cioè solo $1 / 5$ delle giornate

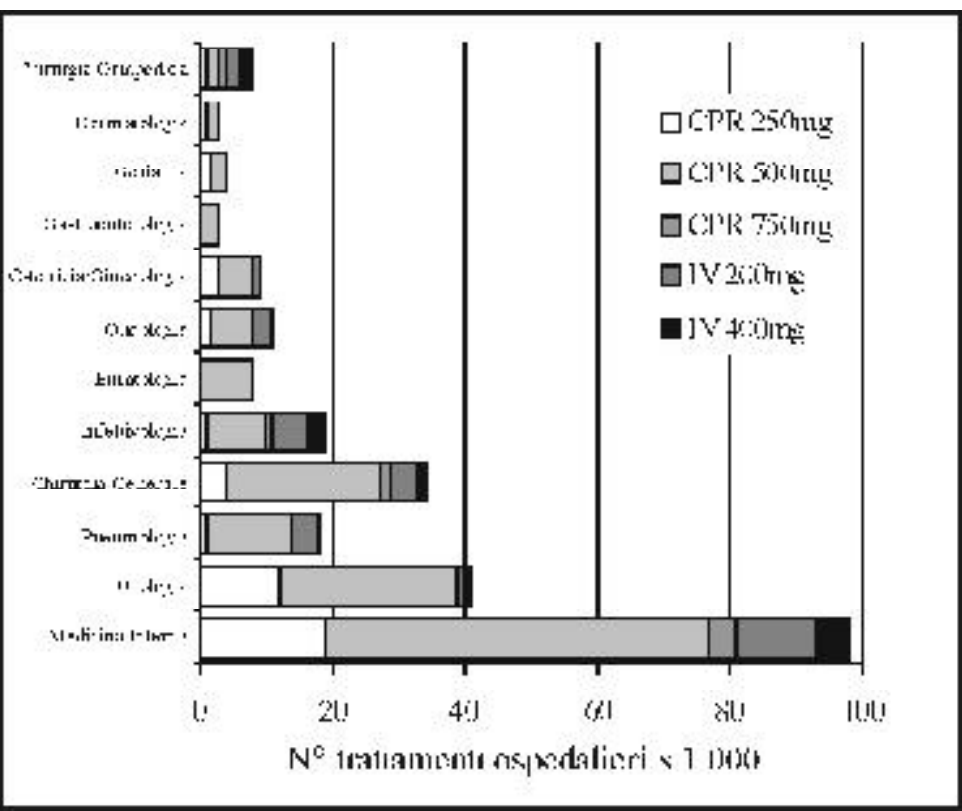

Figura 3.

Distribuzione del numero di trattamenti con ciprofloxacina effettuati nei diversi reparti degli ospedali italiani durante l'anno 2000 (Elaborato da dati AMR, 2000'1).

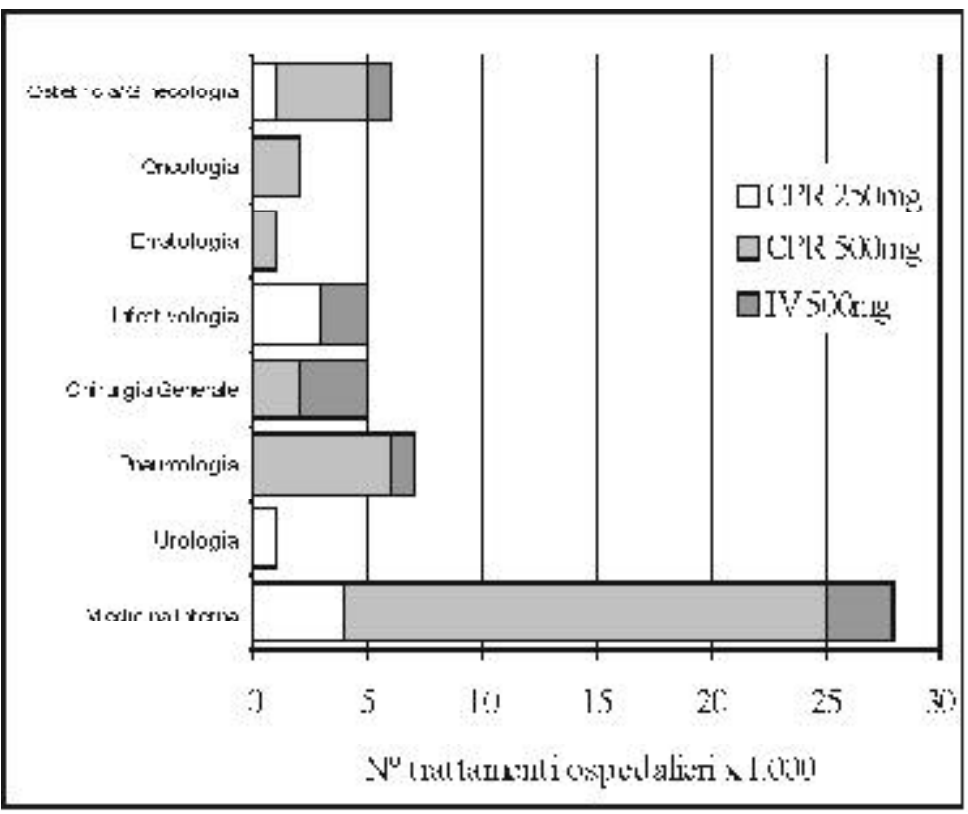

Figura 4.

Distribuzione del numero di trattamenti con levofloxacina effettuati nei diversi reparti degli ospedali italiani durante l'anno 2000 (Elaborato da dati AMR, $2000^{1}$ ). 
di terapia con ciprofloxacina. Anche con la levofloxacina le giornate di terapia parenterale sono circa $1 / 4$ del totale.

Le Figure 5 e 6 riportano le ripartizioni percentuali delle giornate di terapia consumate rispettivamente con ciprofloxacina e con levofloxacina, suddivisi in base alla forma farmaceutica utilizzata ed al livello dose.

La ciprofloxacina per via orale viene somministrata mediamente due volte al giorno, con poche eccezioni in più e soprattutto in meno. Tale comportamento prescrittivo è abbastanza uniforme nei diversi reparti ospedalieri (Tabella 1).

Per levofloxacina la media del numero di

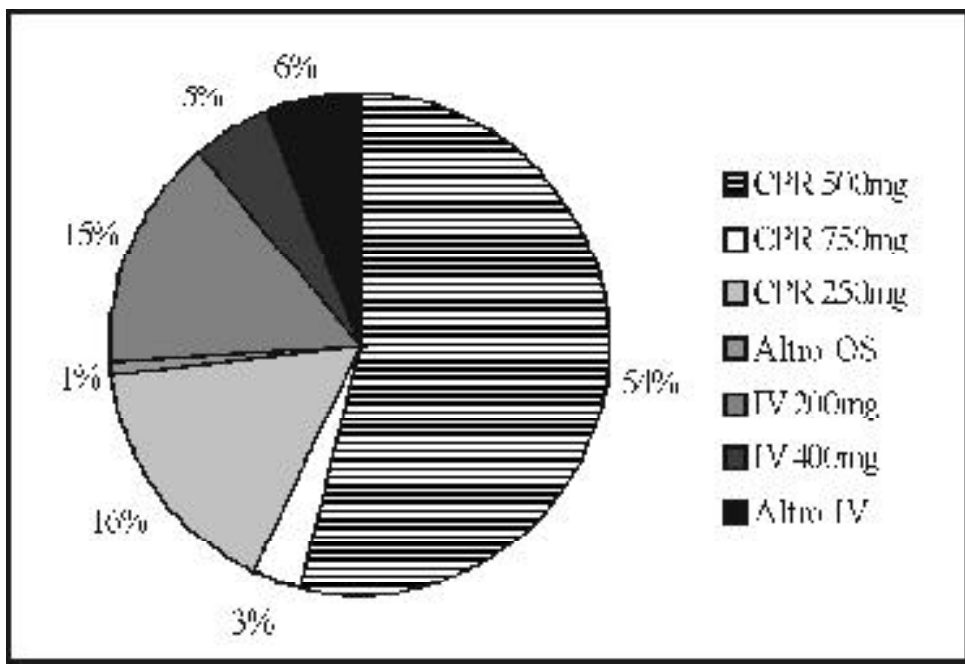

Figura 5.

Distribuzione percentuale del numero di giornate di terapia consumate durante l'anno 2000 negli ospedali italiani con le diverse forme di ciprofloxacina: "altro" indica i casi in cui il trattamento è stato effettuato con più forme farmaceutiche (Elaborato da dati AMR, 2000').

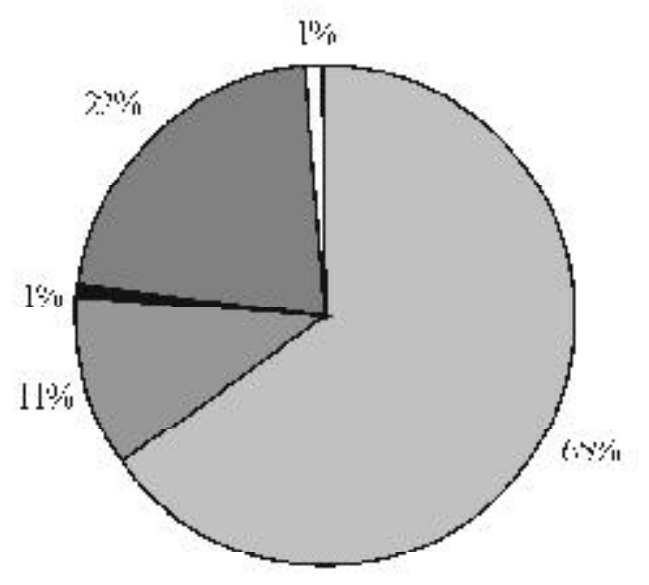

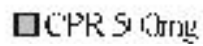

口CI'R 25SLщ

Allm os

口त) 5 ilom

口A $11 \mathrm{~m} \Gamma \mathrm{V}$

Figura 6.

Distribuzione percentuale del numero di giornate di terapia consumate durante l'anno 2000 negli ospedali italiani con le diverse forme di levofloxacina: "altro" indica i casi in cui il trattamento è stato effettuato con più forme farmaceutiche (Elaborato da dati AMR, 2000'1). somministrazioni oscilla attorno al valore di 1,2-1,4 (Tabella 1).

La ciprofloxacina anche per via parenterale viene somministrata più frequentemente due volte al giorno. (Tabella 2).

La levofloxacina parenterale viene somministrata una o due volte al giorno; in particolare, nei pazienti trattati nei reparti oncologici, si utilizza la somministrazione BID (Tabella 2).

La durata del trattamento orale con ciprofloxacina e con levofloxacina varia da una media di 2-3 giorni ad una media di 10-11 giorni (Tabella 3). Le differenze più marcate sono tra i diversi reparti ospedalieri e quindi dipendono dal tipo di infezione da trattare. La durata media del trattamento orale varia anche in relazione alla dose dell'unità farmaceutica.

Un dato da sottolineare è una durata inferiore del trattamento con ciprofloxacina sia nei reparti di Medicina Interna, responsabili del maggiore consumo di flurochinoloni in assoluto, sia in quelli di ematologia dove il trattamento viene attuato frequentemente in pazienti fortemente immunocompromessi.

La durata del trattamento parenterale con ciprofloxacina e con levofloxacina varia da una media di 1 giorno ad una media di 10-11 giorni (Tabella 4). I trattamenti di un giorno, più frequenti nei reparti di ostetricia e ginecologia, sono verosimilmente dovuti a profilassi chirurgica. I trattamenti di pochi giorni sono probabilmente dovuti a quei pazienti che dopo una prima fase per via parenterale passano al trattamento orale (switch therapy).

In questo contesto vanno sottolineati i dati più favorevoli a ciprofloxacina nei reparti di Medicina interna e di Pneumologia.

In conclusione, la ciprofloxacina continua ad essere il fluorochinolone più utilizzato, sia per via orale sia per via parenterale, negli ospedali italiani. Nell'anno 2000 la terapia con levofloxacina era cinque volte meno frequente della terapia con ciprofloxacina.

Infatti, le caratteristiche antibatteriche di ciprofloxacina (ampiezza dello spettro) e conseguentemente l'ampia gamma di indicazioni terapeutiche ne consentono un utilizzo più diffuso, in grado di soddisfare esigenze specifiche di specifici reparti ospedalieri.

Relativamente all'utilizzo dei due farmaci, si nota tendenzialmente un comportamento standard per ciprofloxacina, con due somministrazioni giornaliere del farmaco indipendentemente dalla criticità del rischio infettivo del reparto; per levofloxacina, invece, la tendenza è quella di raddoppiare la frequenza delle somministrazioni giornaliere laddove più importante è il rischio infettivo (Chirurgia generale, Oncologia). 


\begin{tabular}{|c|c|c|c|c|c|c|c|}
\hline & \multicolumn{4}{|c|}{ Ciprofloxacina Orale } & \multicolumn{3}{|c|}{ Levofloxacina Orale } \\
\hline & Totale & $250 \mathrm{mg}$ & $500 \mathrm{mg}$ & $750 \mathrm{mg}$ & Totale & $250 \mathrm{mg}$ & $500 \mathrm{mg}$ \\
\hline TOTALE REPARTI & 1,98 & 1,99 & 2,00 & 1,89 & 1,20 & 1,30 & 1,19 \\
\hline Medicina Interna & 1,98 & 1,99 & 1,99 & 1,81 & 1,15 & 1,33 & 1,14 \\
\hline Urologia & 1,93 & 2,00 & 1,99 & & 1,20 & 1,25 & 1,00 \\
\hline Pneumologia & 2,00 & 2,00 & 2,00 & & 1,30 & & 1,30 \\
\hline Chirurgia Generale & 1,99 & 1,81 & 2,01 & 2,00 & 1,43 & 2,00 & 1,14 \\
\hline Infettivologia & 2,09 & 2,00 & 2,13 & 2,00 & 1,17 & 1,17 & \\
\hline Ematologia & 2,00 & & 2,00 & & 1,45 & & 1,45 \\
\hline Oncologia & 1,96 & 2,00 & 1,91 & & 1,20 & & 1,20 \\
\hline Ostetricia/Ginecologia & 1,96 & 2,00 & 1,94 & & 1,11 & 1,00 & 1,14 \\
\hline Gastroenterologia & 1,83 & & 2,00 & & & & \\
\hline Geriatria & 1,86 & 2,00 & 1,70 & & 1,00 & & \\
\hline Dermatologia & 2,00 & 2,00 & 2,00 & & & & \\
\hline Chirurgia Ortopedica & 2,00 & 2,00 & 2,00 & 2,00 & & & \\
\hline
\end{tabular}

Tabella 1

Media del numero di somministrazioni giornaliere per via orale (Elaborato da dati AMR, 2000').

\begin{tabular}{|c|c|c|c|c|c|}
\hline & \multicolumn{3}{|c|}{$\begin{array}{l}\text { Ciprofloxacina } \\
\text { Parenterale }\end{array}$} & \multicolumn{2}{|c|}{$\begin{array}{l}\text { Levofloxacina } \\
\text { Parenterale }\end{array}$} \\
\hline & Totale & $200 \mathrm{mg}$ & $400 \mathrm{mg}$ & Totale & $500 \mathrm{mg}$ \\
\hline TOTALE REPARTI & 1,91 & 1,88 & 1,90 & 1,15 & 1,16 \\
\hline Medicina Interna & 1,84 & 1,84 & 2,03 & 1,28 & 1,28 \\
\hline Urologia & 2,00 & 2,00 & 2,00 & & \\
\hline Pneumologia & 1,98 & 2,00 & & 1,00 & 1,00 \\
\hline Chirurgia Generale & 1,93 & 1,86 & 2,00 & 1,21 & 1,21 \\
\hline Infettivologia & 2,05 & 2,10 & 2,00 & 1,00 & 1,00 \\
\hline Oncologia & 1,91 & 2,00 & 2,00 & 2,00 & 2,00 \\
\hline Ostetricia/Ginecologia & 2,00 & 2,00 & & 1,00 & 0,00 \\
\hline Geriatria & 1,00 & & 1,00 & & \\
\hline Chirurgia Ortopedica & 1,82 & 2,00 & 1,00 & 1,00 & 1,00 \\
\hline
\end{tabular}

Tabella 2

Media del numero di somministrazioni giornaliere per via parenterale (Elaborato da dati AMR, $\left.2000^{1}\right)$. 
Tabella 3

Media dei giorni di terapia orale (Elaborato da dati AMR, 2000').

\begin{tabular}{|c|c|c|c|c|c|c|c|}
\hline & \multicolumn{4}{|c|}{ Ciprofloxacina Orale } & \multicolumn{3}{|c|}{ Levofloxacina Orale } \\
\hline & Totale & $250 \mathrm{mg}$ & $500 \mathrm{mg}$ & $750 \mathrm{mg}$ & Totale & $250 \mathrm{mg}$ & $500 \mathrm{mg}$ \\
\hline TOTALE REPARTI & 5,7 & 5,8 & 5,7 & 5,6 & 5,7 & 4,1 & 6,1 \\
\hline Medicina Interna & 5,9 & 6,5 & 5,8 & 5,3 & 6,4 & 6,0 & 6,3 \\
\hline Urologia & 4,3 & 5,0 & 4,2 & & 2,5 & 4,0 & \\
\hline Pneumologia & 8,1 & 10,0 & 8,4 & & 7,8 & & 7,8 \\
\hline Chirurgia Generale & 5,1 & 5,3 & 4,7 & 6,0 & 3,5 & 5,0 & 3,5 \\
\hline Infettivologia & 7,1 & 3,0 & 7,7 & 5,0 & 4,0 & 4,0 & \\
\hline Ematologia & 6,9 & & 6,6 & & 11,0 & & 11,0 \\
\hline Oncologia & 5,0 & 5,5 & 5,7 & & 2,5 & & 2,5 \\
\hline Ostetricia/Ginecologia & 3,3 & 3,7 & 3,2 & & 2,3 & 2,0 & 1,8 \\
\hline Gastroenterologia & 9,7 & & 8,0 & & & & \\
\hline Geriatria & 5,5 & 5,5 & 5,0 & & 3,0 & & \\
\hline Dermatologia & 10,0 & 6,0 & 7,0 & & & & \\
\hline Chirurgia Ortopedica & 6,3 & 6,0 & 5,5 & 6,0 & & & \\
\hline
\end{tabular}

Tabella 4

Media dei giorni di terapia parenterale (Elaborato da dati AMR, 2000').

\begin{tabular}{|c|c|c|c|c|c|}
\hline & \multicolumn{3}{|c|}{$\begin{array}{c}\text { Ciprofloxacina } \\
\text { Parenterale }\end{array}$} & \multicolumn{2}{|c|}{$\begin{array}{c}\text { Levofloxacina } \\
\text { Parenterale }\end{array}$} \\
\hline & Totale & $200 \mathrm{mg}$ & $400 \mathrm{mg}$ & Totale & $500 \mathrm{mg}$ \\
\hline TOTALE REPARTI & 6,5 & 7,4 & 5,8 & 6,5 & 6,8 \\
\hline Medicina Interna & 8,5 & 9,7 & 7,0 & 10,7 & 10,7 \\
\hline Urologia & 2,8 & 3,0 & 2,0 & & \\
\hline Pneumologia & 7,5 & 8,5 & & 10,0 & 10,0 \\
\hline Chirurgia Generale & 4,7 & 5,5 & 3,0 & 4,7 & 4,7 \\
\hline Infettivologia & 8,3 & 8,2 & 6,7 & 7,0 & 7,0 \\
\hline Oncologia & 5,3 & 5,3 & & 5,0 & 5,0 \\
\hline Ostetricia/Ginecologia & 0,0 & 3,0 & & 1,0 & 1,0 \\
\hline Chirurgia Ortopedica & 2,8 & 4,0 & 1,0 & 7,5 & 7,5 \\
\hline
\end{tabular}




\section{COSTI DI TRATTAMENTO}

I costi di trattamento antibiotico ospedaliero sono costituiti da diverse voci: costo di acquisto del farmaco, costo dei materiali eventualmente consumati per la preparazione e la somministrazione dell' antibiotico, costo del lavoro del personale medico ed infermieristico, costo delle reazioni avverse, costo degli esami di monitoraggio.

Il costo di acquisto è quello più immediatamente evidente ma non è scontato che sia sempre quello più importante come peso economico.

Abbiamo stimato i costi ospedalieri di un trattamento orale o parenterale con ciprofloxacina o con levofloxacina, limitandoci a considerare il costo d'acquisto del farmaco, assunto al valore di $50 \%$ del prezzo al pubblico. Nel caso di somministrazione parenterale abbiamo anche considerato i costi dei materiali e del lavoro, assumendo che ogni somministrazione comportasse un onere aggiuntivo complessivo medio di Lire $12.500^{11,12}$.
Le stime sono state attuate partendo dai dati di consumo ospedaliero di ciprofloxacina e di levofloxacina che abbiamo sopra esaminato. I risultati sono riassunti nelle Tabelle 5 e 6 , rispettivamente per i costi di un trattamento orale con ciprofloxacina e con levofloxacina.

Le Tabelle 7 e 8 riportano i costi di trattamento rispettivamente con ciprofloxacina parenterale e con levofloxacina parenterale.

Facendo riferimento ai dati riportati nelle Figure 3 e 4, risulta che i reparti di Medicina Interna sono quelli che utilizzano la maggior quantità sia di ciprofloxacina sia di levofloxacina. La differenza di costo tra un ciclo di trattamento con l'uno piuttosto che con l'altro antibatterico, può avere un impatto significativo nell'economia globale di approvvigionamento del farmaco.

In base alle modalità d'uso rilevate negli ospedali italiani, un ciclo di terapia orale con ciprofloxacina compresse da $250 \mathrm{mg}$ costa all'ospedale da un minimo di Lire $8.700(4,49 €)$ ad un massimo di Lire 29.000 (14,98€). Un ciclo di trattamento, attuato utilizzando le compresse da $500 \mathrm{mg}$, costa da un minimo di Lire 17.590

\begin{tabular}{|c|c|c|c|c|c|c|c|c|c|}
\hline & \multicolumn{3}{|c|}{ 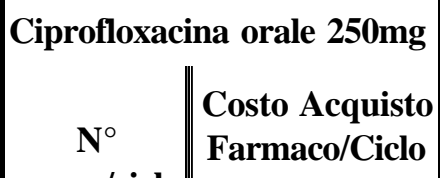 } & \multicolumn{3}{|c|}{ 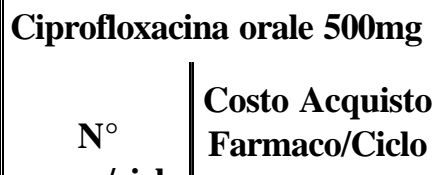 } & \multicolumn{3}{|c|}{$\begin{array}{l}\text { Ciprofloxacina orale } 750 \mathrm{mg} \\
\qquad \begin{array}{c}\text { Costo Acquisto } \\
\mathrm{N}^{\circ}\end{array} \\
\end{array}$} \\
\hline & & Lire & Euro & & Lire & Euro & & Lire & Euro \\
\hline TOTALE REPARTI & 11,5 & 16.649 & 8,60 & 11,4 & 32.415 & 16,74 & 10,6 & 44.585 & 23,03 \\
\hline Medicina Interna & 13,0 & 18.842 & 9,73 & 11,5 & 32.648 & 16,86 & 9,5 & 40.029 & 20,67 \\
\hline Urologia & 10,0 & 14.500 & 7,49 & 8,3 & 23.400 & 12,09 & & & \\
\hline Pneumologia & 20,0 & 29.000 & 14,98 & 16,8 & 47.489 & 24,53 & & & \\
\hline Chirurgia Generale & 9,5 & 13.779 & 7,12 & 9,5 & 26.996 & 13,94 & 12,0 & 50.550 & 26,11 \\
\hline Infettivologia & 6,0 & 8.700 & 4,49 & 16,3 & 46.291 & 23,91 & 10,0 & 42.125 & 21,76 \\
\hline Ematologia & & & & 13,3 & 37.572 & 19,40 & & & \\
\hline Oncologia & 11,0 & 15.950 & 8,24 & 10,8 & 30.686 & 15,85 & & & \\
\hline Ostetricia/Ginecologia & 7,3 & 10.643 & 5,50 & 6,2 & 17.590 & 9,08 & & & \\
\hline Gastroenterologia & & & & 16,0 & 45.336 & 23,41 & & & \\
\hline Geriatria & 11,0 & 15.950 & 8,24 & 8,5 & 24.085 & 12,44 & & & \\
\hline Dermatologia & 12,0 & 17.400 & 8,99 & 14,0 & 39.669 & 20,49 & & & \\
\hline Chirurgia Ortopedica & 12,0 & 17.400 & 8,99 & 11,0 & 31.169 & 16,10 & 12,0 & 50.550 & 26,11 \\
\hline
\end{tabular}

Tabella 5.

Media del numero di somministrazioni di ciprofloxacina orale per ciclo terapeutico e relativi costi ospedalieri di acquisto (anno 2000). 
(9,08 €) ad un massimo di Lire $47.490(24,53 €)$, mentre, se si utilizzano le compresse da $750 \mathrm{mg}$, il costo per ciclo varia da un minimo di Lire $40.000(20,67 €)$ ad un massimo di Lire 50.550 $(26,11 €)$ (Tabella 5).

Il costo medio di un ciclo di terapia orale con levofloxacina compresse da $250 \mathrm{mg}$ varia da un minimo di Lire $6.110(3,16 €)$ ad un massimo di Lire 30.550 (15,78 €). Quando vengono usate le compresse da $500 \mathrm{mg}$ il costo medio di un trattamento orale con levofloxacina varia da un minimo di Lire $9.785(5,05 €)$ ad un massimo di Lire 78.285 (40,40€) (Tabella 6).

Il costo medio d'acquisto della ciprofloxacina parenterale per un ciclo di trattamento ospedaliero varia da un minimo di Lire $189.300(97,77 €)$ ad un massimo di Lire 561.360 (289,92 €), qualora vengano usati i flaconi da $200 \mathrm{mg}$. Il costo medio d'acquisto varia da un minimo di Lire $68.950(35,61 €)$ ad un massimo di Lire 979.780 (506,01€), se vengono impiegati i flaconi da $400 \mathrm{mg}$. C'è tuttavia da considerare che quest'ultimo dosaggio, riservato solo ai casi più gravi, rappresenta oggi il $5 \%$ dell'utilizzo ospedaliero di ciprofloxacina come riportato nella Figura 1 relativa al consumo del farmaco.

Il costo medio d' acquisto della levofloxacina parenterale, nell'unica confezione da $500 \mathrm{mg}$, varia da un minimo di Lire $339.010(175,09 €)$ ad un massimo di Lire $819.390(423,18 €)$ per un ciclo di trattamento ospedaliero (Tabella 7).

Il costo dei materiali e del lavoro varia da un minimo di Lire $12.500(6,47 €)$ per una singola somministrazione ad un massimo di oltre Lire $222.000(114,47 €)$ per un ciclo di trattamento di oltre 14 giorni (Tabella 8).

I costi superiori per levofloxacina rispetto a ciprofloxacina si riscontrano sia presso i repar- ti a elevato utilizzo di fluorochinoloni (Medicina Interna) sia in reparti dove il trattamento è riservato a pazienti molto gravi (Oncologia ad esempio), dove può essere necessario il ricorso alla somministrazione BID di levofloxacina.

L'analisi economica fin qui condotta è indicativa dei livelli di costo attribuibili alle due diverse opzione terapeutiche e alle diverse modalità di somministrazione del trattamento. Questi dati, quindi, rappresentano una base di partenza per valutazioni farmacoeconomiche più esaustive, nelle quali vengano introdotte altre variabili più propriamente cliniche e gestionali: outcome (guarigione clinica, microbiologica, mortalità), schemi terapeutici (es. 400 mg di ciprofloxacina BID e $500 \mathrm{mg}$ di levofloxacina BID nel paziente con infezioni molto gravi), gestione degli eventi avversi, utilizzo di risorse (laboratorio di microbiologia, costo del personale sanitario e non sanitario) ecc.

Infatti, su tale casistica non sono riportati i dati relativi agli esiti terapeutici e quindi non possiamo attuare una valutazione costo/efficacia. Pertanto, non potendo assumere con sufficiente confidenza l'ipotesi di equivalenza terapeutica tra livelli dose analoghi dei due fluorochinoloni, non possiamo interpretare i costi stimati in chiave di minimizzazione dei costi.

\section{PRESSIONE SELETTIVA \\ DELL'ANTIBATTERICO E RESISTENZE IN PSEUDOMONAS AERUGINOSA. DATI STATUNITENSI}

Le infezioni da Pseudomonas aeruginosa rappresentano uno dei problemi più critici in ambito infettivologico essenzialmente perché
Tabella 6

Media del numero di somministrazioni di levofloxacina orale per ciclo terapeutico e relativi costi ospedalieri di acquisto (anno 2000).

\begin{tabular}{|c|c|c|c|c|c|c|}
\hline & \multicolumn{3}{|c|}{ Levofloxacina orale $250 \mathrm{mg}$} & \multicolumn{3}{|c|}{ Levofloxacina orale $500 \mathrm{mg}$} \\
\hline & \multirow{2}{*}{$\begin{array}{c}\mathbf{N}^{\circ} \\
\text { somm/ciclo }\end{array}$} & \multicolumn{2}{|c|}{$\begin{array}{l}\text { Costo Acquisto } \\
\text { Farmaco/Ciclo }\end{array}$} & \multirow{2}{*}{$\begin{array}{c}\mathbf{N}^{\circ} \\
\text { somm/Ciclo }\end{array}$} & \multicolumn{2}{|c|}{$\begin{array}{l}\text { Costo Acquisto } \\
\text { Farmaco/Ciclo }\end{array}$} \\
\hline & & Lire & Euro & & Lire & Euro \\
\hline TOTALE REPARTI & 5,3 & 16.323 & 8,43 & 7,3 & 35.839 & 18,51 \\
\hline Medicina Interna & 8,0 & 24.379 & 12,59 & 7,2 & 35.395 & 18,28 \\
\hline Urologia & 5,0 & 15.275 & 7,89 & & & \\
\hline Pneumologia & & & & 10,2 & 49.928 & 25,79 \\
\hline Chirurgia Generale & 10,0 & 30.550 & 15,78 & 4,0 & 19.571 & 10,11 \\
\hline Infettivologia & 4,7 & 14.297 & 7,38 & & & \\
\hline Ematologia & & & & 16,0 & 78.235 & 40,40 \\
\hline Oncologia & & & & 3,0 & 14.715 & 7,60 \\
\hline Ostetricia/Ginecologia & 2,0 & 6.110 & 3,16 & 2,0 & 9.785 & 5,05 \\
\hline
\end{tabular}


questo Gram-negativo è sensibile solo a un limitato numero di antibiotici (carbapenemici, monobattamici, ceftazidime e ciprofloxacina), e l'emersione di ceppi resistenti è abbastanza frequente. Inoltre, vengono colpiti pazienti in precarie condizioni di salute come i soggetti ricoverati in Terapia Intensiva o affetti da fibrosi cistica.

Recentemente, al fine di indagare in modo più approfondito le correlazioni tra resistenze di $P$. aeruginosa a ciprofloxacina e impatto dei chinolonici in ambito microbiologico, si sono condotti, negli USA, studi sistematici.
Un primo studio condotto al Northwestern Memorial Hospital di Chicago ha valutato l'impatto dell'utilizzo nosocomiale di due chinoloni, ciprofloxacina e ofloxacina, sul profilo delle resistenze di Pseudomonas aeruginosa ${ }^{13}$.

L'ipotesi del lavoro scaturisce dall'idea che la resistenza possa almeno in parte essere dovuta all'uso di chinolonici non così attivi come ciprofloxacina verso $P$. aeruginosa .

L'utilizzo dei due farmaci in quella struttura ospedaliera è riportato nella Figura 7.

L'introduzione nel formulario ospedaliero, e quindi nell'uso clinico, di un fluorochinolone

\begin{tabular}{|c|c|c|c|c|c|c|c|}
\hline \multicolumn{8}{|c|}{ Ciprofloxacina Parenterale 200 mg } \\
\hline \multirow{2}{*}{\multicolumn{2}{|c|}{$\begin{array}{r}\mathrm{N}^{\circ} \\
\text { somm/ciclo }\end{array}$}} & \multicolumn{2}{|c|}{$\begin{array}{l}\text { Costo Acquisto } \\
\text { Farmaco/Ciclo }\end{array}$} & \multicolumn{2}{|c|}{$\begin{array}{l}\text { Costi Materiali e } \\
\text { Lavoro/ciclo }\end{array}$} & \multicolumn{2}{|c|}{ Totale Costo/Ciclo } \\
\hline & & Lire & Euro & Lire & Euro & Lire & Euro \\
\hline TOTALE REPARTI & 13,9 & 438.924 & 226,69 & 173.900 & 89,81 & 612.824 & 316,50 \\
\hline Medicina Interna & 17,8 & 561.363 & 289,92 & 222.410 & 114,87 & 783.773 & 404,78 \\
\hline Urologia & 6,0 & 189.300 & 97,77 & 75.000 & 38,73 & 264.300 & 136,50 \\
\hline Pneumologia & 17,0 & 536.350 & 277,00 & 212.500 & 109,75 & 748.850 & 386,75 \\
\hline Chirurgia Generale & 10,2 & 322.757 & 166,69 & 127.875 & 66,04 & 450.632 & 232,73 \\
\hline Infettivologia & 17,2 & 543.291 & 280,59 & 215.250 & 111,17 & 758.541 & 391,75 \\
\hline Oncologia & 10,7 & 336.323 & 173,70 & 133.250 & 68,82 & 469.573 & 242,51 \\
\hline Ostetricia/Ginecologia & 6,0 & 189.300 & 97,77 & 75.000 & 38,73 & 264.300 & 136,50 \\
\hline \multirow[t]{4}{*}{ Chirurgia Ortopedica } & 8,0 & 252.400 & 130,35 & 100.000 & 51,65 & 352.400 & 182,00 \\
\hline & \multicolumn{7}{|c|}{ Ciprofloxacina Parenterale 400 mg } \\
\hline & \multirow{2}{*}{$\begin{array}{r}\mathrm{N}^{\circ} \\
\text { Somm/ciclo }\end{array}$} & \multicolumn{2}{|c|}{$\begin{array}{l}\text { Costo Acquisto } \\
\text { Farmaco/Ciclo }\end{array}$} & \multicolumn{2}{|c|}{$\begin{array}{l}\text { Costi Materiali } \\
\text { e Lavoro/ciclo }\end{array}$} & \multicolumn{2}{|c|}{$\begin{array}{c}\text { Totale } \\
\text { Costo/Ciclo }\end{array}$} \\
\hline & & Lire & Euro & Lire & Euro & Lire & Euro \\
\hline TOTALE REPARTI & 11,0 & 758.519 & 391,74 & 137.513 & 71,02 & 896.031 & 462,76 \\
\hline Medicina Interna & 14,2 & 979.780 & 506,01 & 177.625 & 91,74 & 1.157 .405 & 597,75 \\
\hline Urologia & 4,0 & 275.800 & 142,44 & 50.000 & 25,82 & 325.800 & 168,26 \\
\hline Chirurgia Generale & 6,0 & 413.700 & 213,66 & 75.000 & 38,73 & 488.700 & 252,39 \\
\hline Infettivologia & 13,3 & 919.793 & 475,03 & 166.750 & 86,12 & 1.086 .543 & 561,15 \\
\hline Chirurgia Ortopedica & 1,0 & 68.950 & 35,61 & 12.500 & 6,46 & 81.450 & 42,07 \\
\hline
\end{tabular}

Tabella 7

Media del numero di somministrazioni di ciprofloxacina parenterale per ciclo terapeutico e relativi costi ospedalieri di acquisto (anno 2000). 
antipseudomonas "sub-ottimale", come ofloxacina, ha contribuito ad aumentare la percentuale di ceppi di P. aeruginosa resistenti ai fluorochinolonici. Il passaggio all'utilizzo del fluorochinolonico più potente, invece, ha avuto l'effetto di far invertire questa tendenza(Figura 8)

Questo studio dimostra una elevata e negativa correlazione tra uso di ofloxacina e attività antipseudomonas della stessa, correlazione negativa in grado di inficiare anche l'attività di ciprofloxacina.

Una indagine simile a quella condotta da Peterson e collaboratori è stata condotta su più ampia scala con una integrazione di tipo farmacoeconomico. Il lavoro, pubblicato nel $1999^{14}$ è stato oggetto anche di una comunicazione congressuale con dati più aggiornati ${ }^{15}$.

I dati forniti dai 145 ospedali partecipanti allo studio erano la spesa per i fluorochinoloni e il tasso di sensibilità a ciprofloxacina dei ceppi di Pseudomonas aeruginosa.

Analizzando i dati dal 1994 al 1997, gli Autori hanno trovato una associazione positiva tra aumento della spesa per acquistare levofloxacina e ofloxacina e riduzione della sensibilità di $P$. aeruginosa ai fluorochinolonici; il risultato, atteso tra l'altro, è la diretta conseguenza dell'utilizzo di farmaci ad attività subottimale verso P. aeruginosa. Levofloxacina e ofloxacina, infatti, avendo una minore efficacia battericida rispetto a ciprofloxacina verso Pseudomonas aeruginosa hanno contribuito significativamente alla selezione di ceppi resistenti. Vice versa, quando si è valutata la correlazione tra spesa (cioè utilizzo) per ciprofloxacina e selezione di ceppi resistenti, il risultato è stato negativo.

Un altro studio che conferma le precedenti osservazioni è stato presentato recentemente al $2^{\text {nd }}$ International Meeting on Antimicrobial Chemotherapy in Clinical Practice ${ }^{16}$.

In questo studio, un più sostenuto uso di levofloxacina (da meno del $2 \%$ a più del $22 \%$ ) ha comportato una riduzione di sensibilità di $P$. aeruginosa dell' $11 \%$ (dall' 82 al 71\%). Successivamente, levofloxacina è stata cancellata dal formulario ospedaliero, e la sensibilità di Pseudomonas aeruginosa a ciprofloxacina aumentava di un $6 \%$.

Quindi, utilizzare l'antibatterico più efficace verso $P$. aeruginosa, comporta indubbi vantaggi sia nell' economia microbiologica dell'ambiente nosocomiale, sia nel vantaggio economico ad esso associato.

\section{COSTO/EFFICACIA}

A completamento dei dati economici riportati, per le analisi costo-efficacia è possibile far riferimento ai dati della letteratura internazionale.

Una valutazione farmacoeconomica relativa all'utilizzo di ciprofloxacina o dell'associazione imipenem/cilastatina nelle polmoniti gravi (nosocomiali o acquisite in comunità) è stata condotta presso il Kern Medical Center di Bakersfield (USA) ${ }^{17}$. Lo studio considerava pazienti $(n=32)$ arruolati nell'ambito di un trial multicentrico disegnato per un confronto clini-

\begin{tabular}{|lc|cc|cc||cc|}
\hline & \multicolumn{7}{c|}{ Levofloxacina Parenterale 500 mg } \\
& $\begin{array}{l}\mathbf{N}^{\circ} \\
\text { somm/ciclo }\end{array}$ & \multicolumn{2}{|c||}{$\begin{array}{c}\text { Costo Acquisto } \\
\text { Farmaco/Ciclo }\end{array}$} & \multicolumn{2}{c|}{$\begin{array}{c}\text { Costi Materiali e } \\
\text { Lavoro/ciclo }\end{array}$} & Totale & Costo/Ciclo \\
\hline \hline & & Lire & Euro & Lire & Euro & Lire & Euro \\
\hline \hline TOTALE REPARTI & $\mathbf{7 , 9}$ & $\mathbf{4 7 4 . 6 3 2}$ & $\mathbf{2 4 5 , 1 3}$ & $\mathbf{9 8 . 8 9 0}$ & $\mathbf{5 1 , 0 7}$ & $\mathbf{5 7 3 . 5 2 2}$ & $\mathbf{2 9 6 , 2 0}$ \\
\hline \hline Medicina Interna & 13,7 & 819.388 & 423,18 & 170.720 & 88,17 & 990.108 & 511,35 \\
\hline Pneumologia & 10,0 & 599.950 & 309,85 & 125.000 & 64,56 & 724.950 & 374,41 \\
\hline Chirurgia Generale & 5,7 & 339.014 & 175,09 & 70.634 & 36,48 & 409.647 & 211,57 \\
\hline Infettivologia & 7,0 & 419.965 & 216,89 & 87.500 & 45,19 & 507.465 & 262,08 \\
\hline Oncologia & 10,0 & 599.950 & 309,85 & 125.000 & 64,56 & 724.950 & 374,41 \\
\hline Chirurgia Ortopedica & 7,5 & 449.963 & 232,39 & 93.750 & 48,42 & 543.713 & 280,80 \\
\hline
\end{tabular}

\section{Tabella 8.}

Media del numero di somministrazioni di levofloxacina parenterale per ciclo terapeutico e relativi costi ospedalieri di acquisto (anno 2000). 
co tra i due trattamenti antibatterici; lo studio multicentrico, peraltro, dimostrava la superiore efficacia clinica di ciprofloxacina rispetto all'antibiotico di confronto (risposta clinica $69 \%$ vs $56 \% ; \mathrm{p}=0,021)^{18}$.

Cinque casi erano esclusi dalla valutazione farmacoeconomica poiché il trattamento antibatterico era stato condotto per meno di 3 giorni. I ventisette pazienti analizzati $(n=13$ ciprofloxacina $400 \mathrm{mg}$ e.v. ogni $8 \mathrm{~h}$; $\mathrm{n}=14$ imipenem/cilastatina) erano equamente distribuiti nei due gruppi di trattamento relativamente alle caratteristiche demografiche e mediche.

Oltre alla valutazione clinica durante la fase di ospedalizzazione, questi pazienti venivano seguiti fino a 37 giorni dopo il trattamento per l'eventuale sviluppo di altre infezioni (persistenza, sovrainfezione o nuova infezione).

Per la valutazione economica, oltre ai costi generati dal trattamento antibatterico, era considerato un costo giornaliero di degenza ospedaliera pari a 1.600 US\$. Gli outcome della ricerca erano il costo per guarigione e il costo totale per paziente.

Da un punto di vista clinico e batteriologico, analogamente a quanto risultato dallo studio multicentrico, anche in questo sottogruppo di pazienti ciprofloxacina con un $77 \%$ di risposte cliniche risultava più efficace di imipenem/ cilastatina $(50 \%)$. L'eradicazione batterica, ugualmente era più favorevole nel gruppo trattato con il fluorochinolone ( $85 \%$ vs $57 \%$ ).

Durante il follow-up, il 38\% dei pazienti che aveva ricevuto ciprofloxacina sviluppò un ulteriore episodio infettivo necessitante di un trattamento antibatterico. Nel gruppo imipenem/ cilastatina questo stesso valore era più negativo (64\%). Questi risultati, che confermano il ruolo fondamentale di ciprofloxacina nel trattamento delle polmoniti gravi, nella valutazione complessiva farmacoeconomica acquisiscono un ruolo determinante essendo il ricovero ospedaliero, piuttosto che il costo della terapia, la voce di spesa più consistente. I costi calcolati in questo studio farmacoeconomico sono riportati nella Tabella 9.

Nel trattamento ospedaliero delle polmoniti, ciprofloxacina rispetto a imipenem/ cilastatina ha dimostrato una eradicazione batterica iniziale superiore e un più basso tasso di infezioni persistenti, sovrainfezioni, recidive e reinfezioni. I costi aggiuntivi nel gruppo trattato con imipenem/cilastatina sono da attribuire, oltre alla superiorità numerica, anche alla maggiore gravità delle stesse: infatti ben il $50 \%$ dei soggetti trattati con imipenem/ cilastatina (7 casi su 14) durante il follow-up sviluppava una infezione (persistente o ricorrente) sostenuta da Pseudomonas aeruginosa, contro un solo caso su $13(7,6 \%)$ del gruppo trattato con ciprofloxacina.

Un ulteriore studio farmacoeconomico ${ }^{19}$ in pazienti ospedalizzati con polmonite moderata e grave (analisi retrospettiva di costo-efficacia dalla prospettiva delle istituzioni) è stato condotto, su base multicentrica in 7 ospedali canadesi. I pazienti erano randomizzati a uno dei seguenti trattamenti: ciprofloxacina -terapia sequenziale- e.v. os; ceftazidime e.v. Il passaggio al trattamento orale con ciprofloxacina era a discrezione dello sperimentatore. La durata del trattamento era vincolata alla risposta clinica e microbiologica. Tutti i pazienti ricevevano anche clindamicina $600 \mathrm{mg}$ e.v. ogni 6 ore la cui somministrazione poteva essere sospesa a discrezione dello sperimentatore. In caso di polmonite da batteri atipici, la clindamicina era sostituita con eritromicina $500 \mathrm{mg}$ e.v. ogni 6 ore e il paziente considerato come "fallimento".

L'analisi farmacoeconomica di questo stu-

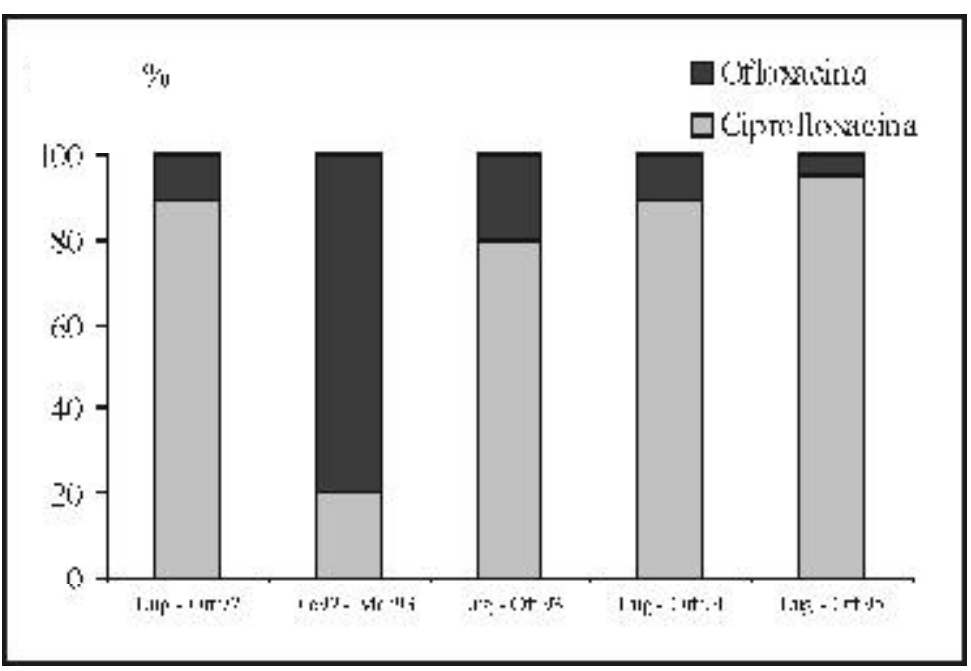

Figura 7

Uso di fluorochinoloni al Northwestern Memorial Hospital di Chicago $^{13}$

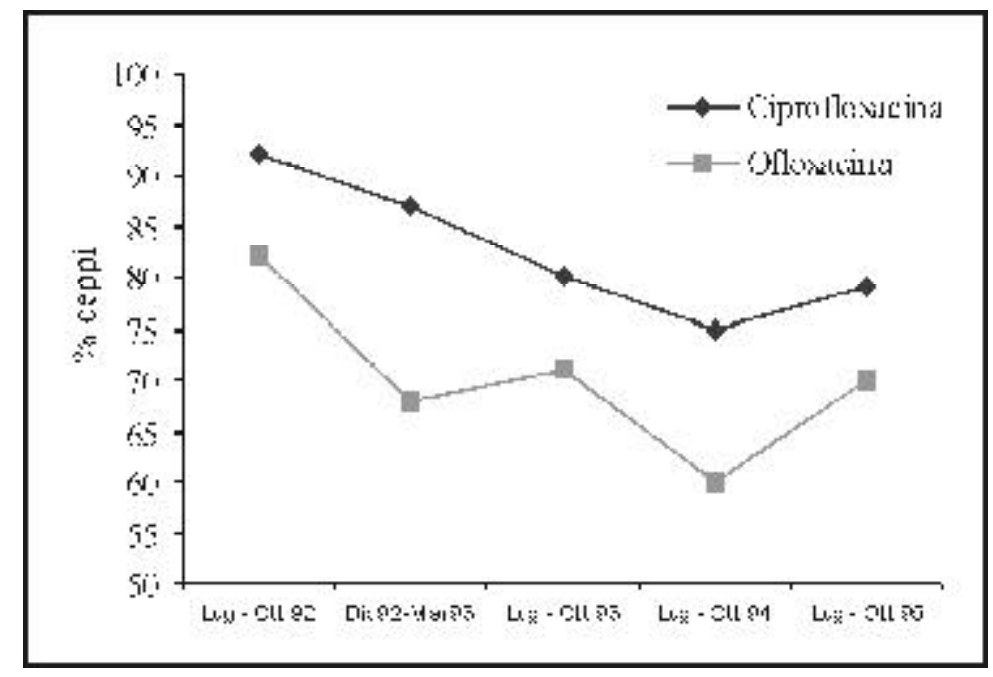

Figura 8

Percentuale di ceppi di Pseudomonas aeruginosa sensibili all'azione antibatterica dei due fluorochinoloni ${ }^{13}$ 
dio ha considerato i costi al livello II, cioè i costi del farmaco e le risorse ancillari correlate all'uso dello stesso (approvvigionamento, allestimento, somministrazione, monitoraggio, trattamento degli eventi avversi e dei fallimenti terapeutici).

L'efficacia e la tollerabilità dei due trattamenti è risultata sovrapponibile.

Relativamente ai costi, tuttavia, i risultati erano a favore di ciprofloxacina, come riportato in dettaglio nella Tabella 10.

Uno studio statunitense ${ }^{16}$ ha valutato da un punto di vista clinico e farmacoeconomico le implicazioni di un trattamento antibatterico in soggetti con infiltrato polmonare ricoverati in Unità di Terapia Intensiva. L'uso non strettamente necessario di antibiotici, infatti, è comune in questi pazienti essendo spesso l'infiltrato non correlato a polmonite batterica. Utilizzando quale strumento diagnostico di screening il CPIS (Clinical Pulmonary Infection Score $)^{17}$, i pazienti ricevevano un trattamento con terapia standard (?-lattamine/ inibitori ?-lattamasi; chinoloni; aminoglicosidi; vancomicina) qualora il punteggio CPIS fosse $>6$ (predittivo di polmonite). Per i pazienti con punteggio $<6$ (remote possibilità di polmonite), si procedeva a una randomizzazione con terapia antibiotica standard per 10-21 giorni oppure con ciprofloxacina $400 \mathrm{mg}$ e.v. ogni 8 ore per 3 giorni. In quest'ultimo gruppo di pazienti, in terza giornata, si procedeva ad un'ulteriore valutazione del CPIS; qualora il punteggio fosse rimasto $<6$ si sospendeva il trattamento con ciprofloxacina, per punteggi superiori, invece, si instaurava una terapia antibiotica appropriata sulla base dell'esame colturale microbiologico. I risultati clinici si sono dimostrati favorevoli ad un trattamento breve con ciprofloxacina rispetto alla terapia standard poiché un minor numero di soggetti necessita- va di proseguire la terapia antibatterica dopo $\mathrm{i}$ primi 3 giorni (Figura 9).

Un altro dato a favore dell'utilizzo di ciprofloxacina per un breve periodo di tempo in pazienti ricoverati in UTI e con infiltrato polmonare è correlato allo sviluppo di resistenze batteriche e di superinfezioni, come dettagliato nella Tabella 11.

Relativamente all'aspetto economico delle due diverse opzioni terapeutiche, l'utilizzo di ciprofloxacina ha presentato dei vantaggi rispetto alla terapia standard. Fissando come limite temporale del confronto i primi 3 giorni di trattamento, il costo complessivo della terapia antibatterica di pazienti con infiltrato polmonare (CPIS<6) era di 6.484 US\$ nel gruppo ciprofloxacina, 16.004 US\$ nel gruppo trattato con terapia standard $(\mathrm{p}=0,0001)$.

Passando a un altro settore di utilizzo di ciprofloxacina, quello delle manifestazioni febbrili associate a neutropenia, sono disponibili i dati clinici ed economici di un recente studio canadese $^{22}$.

Presso il centro onco-ematologico della British Columbia University si è condotto uno studio in aperto atto a valutare un programma stepdown correlato all'uso di ciprofloxacina in pazienti con febbre da neutropenia $\left(>38^{\circ} \mathrm{C}\right.$, conta neutrofili $<500 / \mathrm{mm}^{3}$ o maggiore ma in rapido declino, o trattati con chemioterapici inducenti neutropenia).

Nel primo periodo dello studio (primi 6 mesi) si sono analizzate le cartelle cliniche di pazienti trattati con ciprofloxacina $400 \mathrm{mg}$ e.v. ogni 12 ore all'interno di un protocollo di terapia antibatterica comprendente l'associazione con tobramicina e vancomicina oppure con ceftazidime e vancomicina. Nei successivi 6 mesi si applicò la stessa analisi dopo l'introduzione di un protocollo alternativo relativamente al dosaggio di ciprofloxacina (stepdown 400
Tabella 9

Costi di due diverse opzioni terapeutiche per la polmonite grave 17

\begin{tabular}{|lcc|}
\hline Parametro & Ciprofloxacina $(\mathbf{n}=\mathbf{1 3})$ & Imipenem/cilastatina $(\mathbf{n}=\mathbf{1 4})$ \\
\hline \hline Costi totali & 291.269 US\$ & 533.140 US\$ \\
\hline \hline Costi per guarire 1 paziente & 29.127 US\$ & 76.163 US\$ \\
\hline
\end{tabular}

\section{Tabella 10}

Costi del trattamento in dollari canadesi ${ }^{19}$

\begin{tabular}{|lccc|}
\hline & Ciprofloxacina & Ceftazidime & p \\
\hline \hline Costo per paziente $(\$)$ & $1.132 ? 92$ & $1.676 ? 105$ & $<0,0003$ \\
\hline \hline Costo giornaliero $(\$)$ & $89 ? 5$ & $149 ? 4$ & $<0,0001$ \\
\hline
\end{tabular}


mg-200 mg e.v.-e.v. oppure e.v.-os) nei pazienti clinicamente e emodinamicamente stabili, senza febbre da 48 ore, con coltura negativa o positiva per patogeni sensibili all'antibiotico in uso (esclusa Pseudomonas aeruginosa o Enterobacter cloacae). Si optava per lo stepdown e.v.-e.v. nei casi in cui non fosse ipotizzabile un recupero della conta dei neutrofili entro 3-5 giorni e condizioni obiettive quali mucosite o diarrea grave precludessero la somministrazione orale di ciprofloxacina.

La risposta clinica, definita come apiressia e risoluzione completa o parziale di altri segni e sintomi di infezione, risultava sovrapponibile nei due gruppi ( $83 \%$ vs $81 \%$ rispettivamente). Anche la risposta microbiologica (eradicazione, persistenza, colonizzazione, sovrainfezione) non presentava differenze statisticamente significative tra i due gruppi. Il vantaggio di un regime stepdown applicato a ciprofloxacina nel trattamento di pazienti con febbre da neutropenia è quindi di tipo economico; da questo studio, infatti, il costo totale di un ciclo di trattamento con ciprofloxacina che, mediamente, era di 874 \$ canadesi, con l'adozione di una strategia stepdown, si riduceva a 750 \$ canadesi.

Sempre in tema di strategie alternative relative alle modalità di somministrazione di ciprofloxacina, è stata condotta una analisi farmacoeconomica in pazienti con batteriemia da Gram-negativi presso il Montefiore Medical Center di New York ${ }^{23}$.

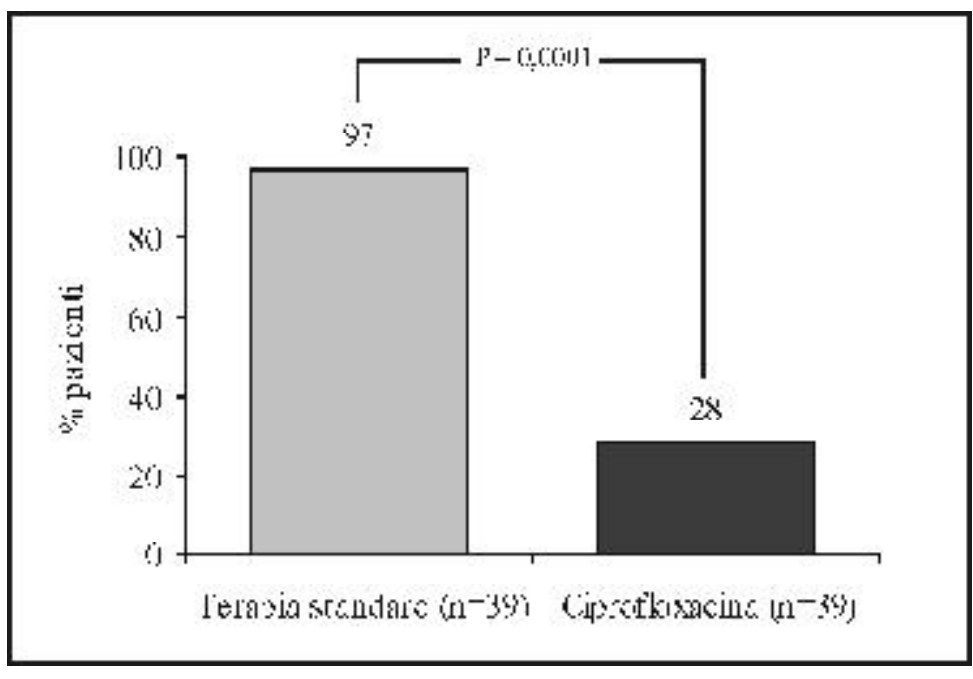

Figura 9

Proseguimento della terapia antibatterica dopo 3 giorni $^{20}$

I pazienti erano randomizzati a un gruppo di trattamento con antibiotici e.v. (generalmente una ?-lattamina e un aminoglicoside) $(n=26)$ oppure a un gruppo che dopo 72 ore di questo stesso regime passava a terapia orale con ciprofloxacina $750 \mathrm{mg}$ ogni 12 ore $(n=24)$. Un terzo gruppo di pazienti con batteriemia da Gram-negativi, non arruolati nello studio, era valutato quale gruppo di controllo $(n=50)$.

I principali parametri valutati nello studio erano la risposta clinica, la tollerabilità dei trattamenti e la durata dell'ospedalizzazione.

\begin{tabular}{|lccc|}
\hline Parametri & $\begin{array}{c}\text { Ciprofloxacina } \\
\mathbf{n}=\mathbf{3 7}\end{array}$ & $\begin{array}{c}\text { Terapia standard } \\
\mathbf{n}=\mathbf{3 7}\end{array}$ & $\mathbf{P}=$ \\
\hline Resistenza batterica e/o superinfezioni & $14 \%$ & $38 \%$ & 0,017 \\
\hline Microrganismi & $8 \%$ & $16 \%$ & \\
\hline Pseudomonas aeruginosa & - & $5 \%$ \\
\hline Enterobacter cloacae & $5 \%$ & $14 \%$ \\
\hline Staph. aureus meticillino resistente $($ MRSA) & $3 \%$ & - \\
\hline Pseudomonas cepacia & - & $3 \%$ \\
\hline Citrobacter freundii & - & $3 \%$ \\
\hline Pseudomonas stutzeri & $3 \%$ & $11 \%$ \\
\hline Enterococcus spp & 1 & 3 \\
\hline E. faecalis $(n)$ & 0 & $14 \%$ \\
\hline E. faecium vancomicino-resistente $(n)$ & $8 \%$ & 3 \\
\hline Candida spp & 3 & 2 \\
\hline C. albicans $(n)$ & 0 & \\
\hline \hline C. glabrata $(n)$ & & \\
\hline
\end{tabular}

Tabella 11

Sviluppo di resistenze batteriche elo superinfezioni ${ }^{20}$ 


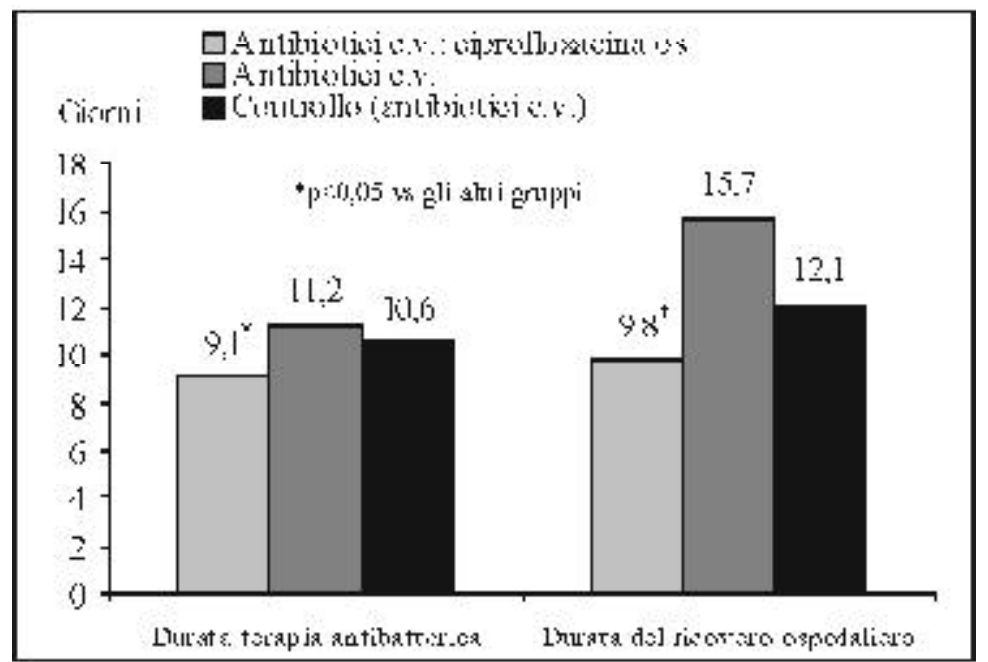

Figura 10

Durata della terapia e dell'ospedalizzazione $e^{23}$

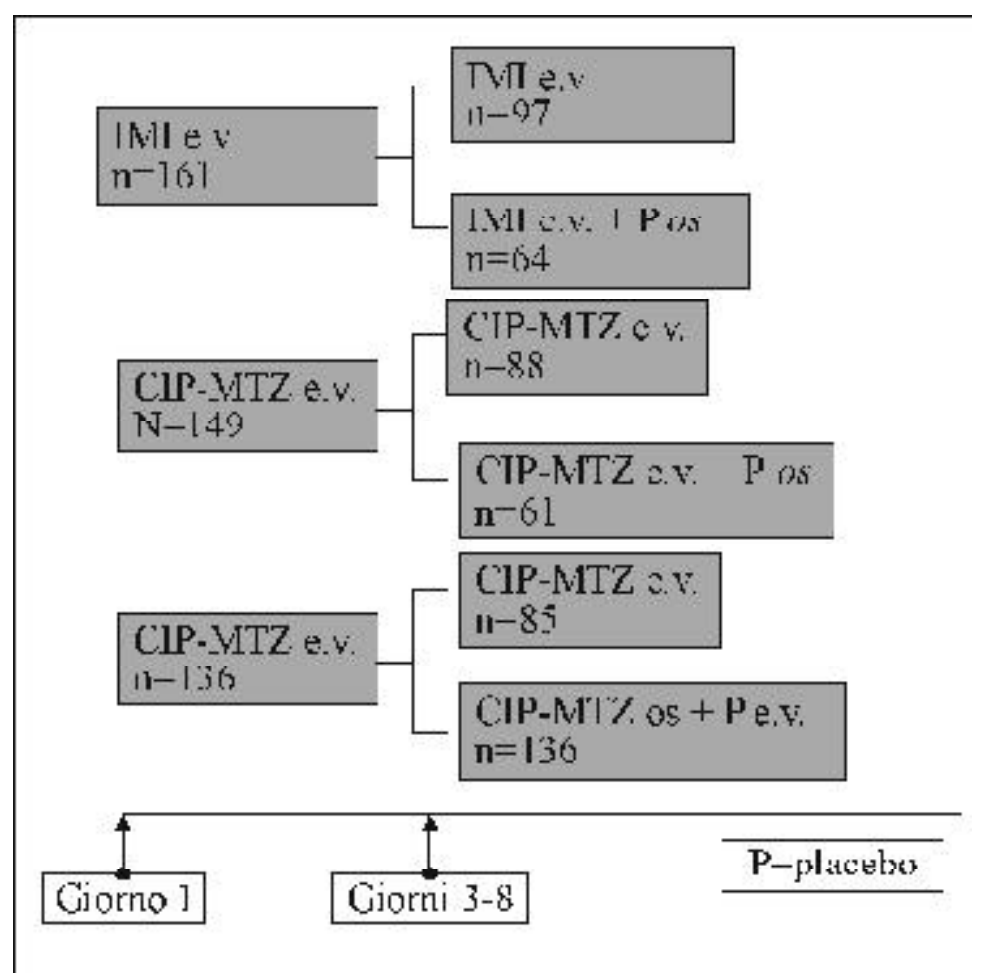

Figura 11.

Schema di trattamento ${ }^{24}$
La risoluzione della batteriemia si otteneva nell' $83 \%$ dei pazienti trattati con la terapia sequenziale e.v. e ciprofloxacina os, nel $77 \%$ dei pazienti trattati con terapia e.v. e nel $77 \%$ del gruppo di controllo. La tollerabilità risultava buona per tutti i trattamenti. Differenze significative, invece, erano presenti relativamente alla durata del trattamento antibiotico e alla durata dell'ospedalizzazione, come riportato nella Figura 10.

Relativamente alla valutazione economica, la riduzione della durata dell'ospedalizzazione comportava un risparmio, nel gruppo trattato con terapia sequenziale antibiotici e.v./ ciprofloxacina os, di oltre 78.000 US\$.

Una indagine farmacoeconomica con ciprofloxacina ha coinvolto anche pazienti con infezione intra-addominale $(n=671)$ trattati secondo tre diversi protocolli in 22 centri nordamericani ${ }^{24}$. Il disegno dello studio era in doppio cieco, randomizzato.

Lo schema di trattamento, riportato in Figura 11, prevedeva l'utilizzo dei seguenti farmaci: ciprofloxacina $400 \mathrm{mg}$ e.v. ogni 12 ore e metronidazolo $500 \mathrm{mg}$ e.v. ogni 6 ore (CIP-MTZ e.v.); ciprofloxacina $500 \mathrm{mg}$ os ogni 12 ore e metronidazolo $500 \mathrm{mg}$ os ogni 6 ore (CIP-MTZ os); imipenem/cilastatina $500 \mathrm{mg}$ e.v. ogni 6 ore (IMI e.v.).

Per mantenere le condizioni di cecità era prevista la somministrazione e.v. o orale di placebo (P).

Una sintesi dei risultati clinici e farmacoeconomici di questo studio sono riportati nella Tabella 12.

\section{CONCLUSIONI}

Una gestione corretta delle infezioni batteriche all'interno di una struttura ospedaliera necessita certamente della massima attenzione e competenza per le implicazioni sia mediche sia economiche ad essa correlate.

Innanzi tutto, andrà posta la massima attenzione nella scelta dell'agente antibatterico
Tabella 12

Risultati clinici e dati economici dello stu$\mathrm{dio}^{24}$

\begin{tabular}{||ccccc|}
\hline \hline N. pazienti & Trattamento & Successo clinico & Durata terapia & Costo medio \\
\hline \hline 51 & CIP-MTZ e.v./os & $98 \%$ & 9,1 giorni & 7.678 US\$ \\
\hline 125 & $\begin{array}{c}\text { Terapia e.v }+ \\
\text { placebo } o s\end{array}$ & $94 \%$ & 10,1 giorni & 8.744 US\$ \\
\hline 97 & IMI e.v. & $75 \%$ & 13,8 giorni & 12.418 US\$ \\
\hline \hline 173 & CIP-MTZ e.v. & $77 \%$ & 13,4 giorni & 12.219 US\$ \\
\hline
\end{tabular}


più indicato per una terapia mirata o empirica di quella specifica infezione. I parametri guida, in questo caso sono la conoscenza dei dati microbiologici, farmacocinetici. farmacodinamici e clinici dei vari antinfettivi. Ma oltre a questo, riveste un'importanza altrettanto elevata conoscere la realtà microbiologica "locale" in termini di specie batteriche e sensibilità delle stesse. Va da sé che questi dati andranno poi elaborati in funzione dello stato fisico del paziente necessitante del trattamento antibatterico. Queste considerazioni, che carat- terizzano ogni atto terapeutico, tuttavia, devono essere integrate da una considerazione aggiuntiva: l'antibiotico in uso, infatti, può contribuire a modificare l'ecologia della flora batterica presente nel nosocomio, con la possibilità di selezionare ceppi resistenti.

A tutte queste considerazioni prettamente mediche, infine, c'è da aggiungere anche quella economica, importante per il sociale, ma non determinante per avere i migliori benefici clinici nell'immediato e nel futuro.

\section{BIBLIOGRAFIA}

1) The Hospital Anti-Infective Market. AMR 2000

2) D Bassetti: I chinoloni. In D Bassetti: Chemioterapici antinfettivi e loro impiego razionale. VII edizione. Intramed, Milano 2001. pp181-210

3) D Nathawani et al.: Sequential antimicrobial therapy: the role of quinolones. J Antimicrob Chemother 1997; 39: 441-46

4) A Marchese et al.: Role of global surveillance in combating bacterial resistence. Drugs 2001; 61: 167-73

5) RN Grüneberg et al.: Results of the Alexander Project: a continuing, multicenter study of the antimicrobial susceptibility of community-acquired lower respiratory tract bacterial pathogens. Diagn Microbiol Infect Dis 1996; 25: 169-81

6) FJ Schmitz et al.: Comparative activities of six different fluoroquinolones against 9,682 clinical bacterial isolates from 20 European university hospitals participating in the European SENTRY surveillance programme. The SENTRY participants Groups. Int J Antimicrob Agents 1999; 12: 311-7

7) G Fadda et al.: Osservatorio Epidemiologico Italiano per il monitoraggio delle resistenze agli antibiotici nei patogeni respiratori comunitari 1997-1999. Suggerimenti per la pratica clinica. GIMMOC 2000; 4: 3-58

8) A Lang et al.: Antimicrobial use and susceptibility rates in isolates from intensive care unit and other nosocomial inpatient and outpatients areas. New Microbiol 2001; 24: 47-56

9) JM Mylotte et al.: Epidemiology of nosocomial infection and resistant organisms in patients admitted for the first time to an acute rehabilitation unit. Clin Infect Dis 2000; 30: 425-32

10) De Lalla F, Eandi M. Indagine sull'uso di antibiotici nel trattamento delle infezioni delle basse vie respiratorie negli ospedali italiani: considerazioni farmacoeconomiche. Giornale Italiano Chemioterapia, 1998.

11) Bondonio P, Eandi M. I tempi ed $i$ costi di lavoro associati al trattamento farmacologico: analisi di alcune opportunità di risparmio. Farmeconomia 1995; 3: 36-45

12) Tartaro D, Becchio D, Zara GP, Dirindin N. Il costo dei materiali d'uso e remunerabilità delle diverse linee di prodotto. Farmeconomia 1995; 3: 5-11,

13) LR Peterson et al.: Management offluoroquinolone resistance in Pseudomonas aeruginosa. Outcome of monitored use in a referral hospital. Int J Antimicrob Agents 1998; 10: 207-214 
14) RP Rifenburg et al.: Influence of fluoroquinolone purchasing patterns on antimicrobial expenditures and Pseudomonas aeruginosa susceptibility. Am J Health Syst Pharm 1999: 56: 2217-23

15) SM Bhavnani et al.: Association between fluoroquinolone expenditure and ciprofloxacin susceptibility of $P$. aeruginosa among U.S. hospital. $39^{\text {th }}$ Interscience Conference on Antimicrobial Agents and Chemotherapy (ICAAC), San Francisco 1999, 26-29 Sept. Abs 182

16) PB Iannini et al.: Impact of fluoroquinolone substitution on pseudomonal susceptibility rates and antimicrobial use. Pharmacotherapy 2001; 21: 375S, Abs 111

17) JW Caldwell et al.: Clinical and economic evaluation of subsequent infection following intravenous ciprofloxacin or imipenem therapy in hospitalized patients with severe pneumonia. J Antimicrob Chemother 1999; 43 (Suppl. A): 129-34

18) MP Fink et al.: Treatment of severe pneumonia in hospitalized patients: results of a multicenter, randomized, double blind trial comparing intravenous ciprofloxacin with imipenem-cilastatin. Antimicrob Agents Chemother 1994; 38: 547-57

19) JA Paladino: Pharmacoeconomic comparison of sequential IV/oral ciprofloxacin versus ceftazidime in the treatment of nosocomial pneumonia. Can J Hosp Pharm 1995; 48: 276-83

20) N Singh et al.: Short-course empiric antibiotic therapy for patients with pulmonary infiltrates in the intensive care unit. A proposed solution for indiscriminate antibiotic prescription. Am J Respir Crit Care Med 2000; 162: 505-11

21) J Pugin et al.: Diagnosis of ventilator-associated pneumonia by bacteriologic analysis of bronchoscopic and nonbronchoscopic “blind” bronchoalveolar lavage fluid. Am Rev Respir Dis 1991; 143: 1121-29

22) CA Marra et al:: A new ciprofloxacin stepdown program in the treatment of high-riskfebrile neutropenia: a clinical and economic analysis. Pharmacotherapy 2000; 20: 931-40

23) M Amodio-Groton et al.: Sequential parenteral and oral ciprofloxacin regimen versus parenteral therapy for bacteriemia. A pharmacoeconomics analysis. Ann Pharmacother 1996; 30: 596-602

24) DJ Walters et al.: Cost-effectiveness of ciprofloxacin plus metronidazole versus imipenem-cilastatin in the treatment of intra-abdominal infections. Pharmacoeconomics 1999; 16: 551-61 